\title{
Elucidating the Doping Mechanism in Fluorene-Dithiophene Based Hole Selective Layer Employing Ultra-Hydrophobic Ionic Liquid Dopant
}

Navind Harindu Hemasiri, Samrana Kazim, Laura Calio, Sanghyun Paek, Manuel Salado, Gianluca Pozzi, Luis Lezama, Mohammad Khaja Nazeeruddin, Shahzada Ahmad

Submitted date: $27 / 01 / 2020$ - Posted date: 31/01/2020

Licence: CC BY-NC-ND 4.0

Citation information: Hemasiri, Navind Harindu; Kazim, Samrana; Calio, Laura; Paek, Sanghyun; Salado, Manuel; Pozzi, Gianluca; et al. (2020): Elucidating the Doping Mechanism in Fluorene-Dithiophene Based Hole Selective Layer Employing Ultra-Hydrophobic Ionic Liquid Dopant. ChemRxiv. Preprint. https://doi.org/10.26434/chemrxiv.11734419.v1

Perovskite solar cells have set a new milestone in terms of efficiencies in the thin film photovoltaics category. Long-term stability of perovskite solar cells is of paramount importance but remains a challenging task. The lack of perovskite solar cells stability in real-time operating conditions erodes and impedes commercialization. Further improvements are essential with a view to delivering longer-lasting photovoltaic (PV) performances. An ideal path in this direction will be to identify novel dopants for boosting the conductivity and hole mobility of hole transport materials (HTMs), and by so doing the usage of hygroscopic and deliquescent additive materials can be avoided. Pyridine-based ionic liquids represent a well-known class of ultra-hydrophobic materials, which are suitable for their application in opto-electrical devices. The present work demonstrates the employment of ionic liquids into a dissymmetric fluorene-dithiophene, FDT (2',7' -bis(bis(4-methoxyphenyl)amino) spiro[cyclopenta[2,1-b:3,4-b']dithiophene-4,9'-fluorene]) based HTM to understand the doping mechanisms. N-heterocyclic hydrophobic ionic liquid, 1-butyl-3-methylpyidinium bis(trifluoromethylsulfonyl)imide (BMPyTFSI) as p-type dopant for FDT was found to increase the conductivity of FDT, to higher geometrical capacitance, to facilitate homogeneous film formation, and to enhance device stability. Our findings open up a broad range of hole-transport materials to control the degradation of the underlying water-sensitive active layer by substituting hygroscopic element.

File list (1) 


\title{
Elucidating the Doping Mechanism in fluorene-dithiophene based hole selective layer employing ultra-hydrophobic Ionic Liquid Dopant
}

\author{
Naveen Harindu Hemasiri ${ }^{1}$, Samrana Kazim ${ }^{1,2}$ Laura Calio $^{3}$, Sanghyun Paek ${ }^{4}$, Manuel \\ Salado $^{1}$, Gianluca Pozzi ${ }^{5}$, Luis Lezama ${ }^{6}$, Mohammad Khaja Nazeeruddin ${ }^{4}$ and Shahzada \\ Ahmad $^{1,2}$
}

${ }^{1}$ BCMaterials, Basque Center for Materials, Applications and Nanostructures, Bld. Martina

Casiano, UPV/EHU Science Park, Barrio Sarriena s/n, 48940 Leioa, Spain Tel: +34

946128811 Email:shahzada.ahmad@bcmaterials.net

${ }^{2}$ IKERBASQUE, Basque Foundation for Science, Bilbao, 48013, Spain

${ }^{3}$ Abengoa Research, Abengoa, C/ Energia Solar n ${ }^{\circ}$ 1, Campus Palmas Altas-41014, Sevilla, Spain

${ }^{4}$ Group for Molecular Engineering of Functional Materials, École Polytechnique Fédérale de Lausanne (EPFL), CH-1951 Sion, Switzerland

${ }^{5}$ Consiglio Nazionale delle Ricerche, Istituto di Scienze e Tecnologie Chimiche "Giulio

Natta” (CNR-SCITEC), via Golgi 19, 20133 Milano, Italy

${ }^{6}$ Departamento de Química Inorgánica, Facultad de Ciencia y Tecnología, Universidad del País Vasco, UPV/EHU, Sarriena s/n, 48940 Leioa, Spain

\begin{abstract}
Perovskite solar cells have set a new milestone in terms of efficiencies in the thin film photovoltaics category. Long-term stability of perovskite solar cells is of paramount importance but remains a challenging task. The lack of perovskite solar cells stability in realtime operating conditions erodes and impedes commercialization. Further improvements are essential with a view to delivering longer-lasting photovoltaic (PV) performances. An ideal path in this direction will be to identify novel dopants for boosting the conductivity and hole mobility of hole transport materials (HTMs), and by so doing the usage of hygroscopic and deliquescent additive materials can be avoided. Pyridine-based ionic liquids represent a wellknown class of ultra-hydrophobic materials, which are suitable for their application in optoelectrical devices. The present work demonstrates the employment of ionic liquids into a dissymmetric fluorene-dithiophene, FDT (2',7' -bis(bis(4-methoxyphenyl)amino) spiro[cyclopenta[2,1-b:3,4-b']dithiophene-4,9'-fluorene]) based HTM to understand the
\end{abstract}


doping mechanisms. $N$-heterocyclic hydrophobic ionic liquid, 1-butyl-3-methylpyidinium bis(trifluoromethylsulfonyl)imide (BMPyTFSI) as p-type dopant for FDT was found to increase the conductivity of FDT, to higher geometrical capacitance, to facilitate homogeneous film formation, and to enhance device stability. Our findings open up a broad range of hole-transport materials to control the degradation of the underlying water-sensitive active layer by substituting hygroscopic element.

\section{Introduction}

The fuelling demand for cost-effective and new energy harvesting technologies led to the rapid progress in power conversion efficiency (PCE) of perovskites for solar cells fabrication, since its first report in solid-state configuration in $2012^{1-3}$. In spite of the unprecedented PCE achieved in excess of $25 \%{ }^{1,4}$, the lack of long-term stability of perovskite solar cells (PSCs) under operative conditions impedes their commercialization ${ }^{5,6}$. To fabricate efficient PSCs the role of hole-transporting material (HTM) which can effectively extract positive charges from the active layer (perovskite) and transfer them to the electrode is paramount ${ }^{7,8}$. To date, amorphous 2,2',7,7'-tetrakis( $N, N$-di-4-methoxyphenyl-amine)-9,9'-spirobifluorene (SpiroOMeTAD) is the well-studied and ubiquitous HTM for high performance PSCs fabrication, mainly due to its solubility, molecular orientation and weak absorption in the visible region ${ }^{9}$. However, the pristine Spiro-OMeTAD shows high series resistance and charge recombination in devices due to low carrier-charge mobility ${ }^{9-11}$. Further, laborious purification procedure and demanding synthesis approach hinders its widespread commercial applications ${ }^{9,12}$. This allowed to identify suitable high-performance HTMs as an alternative for state-of-the-art Spiro-OMeTAD and polytriarylamine polymer (PTAA).

Significant efforts have been laid to explore the structure-property relationship for operational stability and enhanced PCEs, which requires short-circuit current densities near the maximum of $\sim 25 \mathrm{~mA} \mathrm{~cm}{ }^{-2}$ simultaneously with high open-circuit voltages and fill factors ${ }^{8,9,12}$. A dissymmetric fluorene-dithiophene based HTM (2',7'-bis(bis(4methoxyphenyl)amino)spiro[cyclopenta[2,1-b:3,4-b']dithiophene-4,9'-fluorene], termed as FDT, was investigated with a remarkable PCE of 20.2\% in PSCs ${ }^{8}$. In contrast with state-ofthe-art Spiro-OMeTAD, FDT bears many distinct merits including ease of synthesis along with modification by molecularly chemical engineering via electron donor-acceptor groups, ability to dissolve in an eco-friendly solvent such as toluene instead of chlorobenzene, and high PV performances ${ }^{8,9,12}$. 
The interaction between HTM and perovskite at the interface occurs mainly among methoxy groups of the HTM and methylammonium sites of the perovskite, which has been systematically described using computational simulation ${ }^{9,13,14}$. FDT has a dissymmetrical core incorporating a fluorene moiety and a cyclopentadithiophene unit, which are connected through a $\mathrm{sp}^{3}$-hybridized carbon. Due to additional thiophene-iodine interactions, FDT shows enhanced interaction with perovskite surface, confirming firm bonding and strong interfacial coupling between FDT and perovskite ${ }^{8,9,15}$. Thus, it creates an alternative hole-transfer route for photogenerated holes from perovskite iodine atoms to the HTM effectively. The presence of electron-rich sulphur heteroatoms in FDT also introduces non-covalent intermolecular interactions that influence the molecular packing of the whole system. Further, the existence of extremely short intermolecular S...S contact between the neighbouring columns in FDT provides better interaction with perovskite, promoting a strongly efficient hole-transport system as compared to Spiro-OMeTAD ${ }^{8,9,15,16}$.

Recently, different types of chemical doping to HTM has been reported to ensure the fabrication of efficient PSCs. For instance, lithium salts are widely used for p-doping which tend to be comprised of molecules with significantly high electron affinities to enhance the hole carrier mobility. Lithium bis(trifluoromethanesufonyl)imide (LiTFSI) is the widely investigated salt employed for chemical doping of HTM, however, its ultra-hygroscopicity promotes moisture absorption to HTM, under atmospheric condition, effecting the degradation of both HTM and the underlying perovskite material ${ }^{7,11}$. 4-tert-Butylpyridine ( $t$ BP) is commonly used as an additive to achieve higher open-circuit voltage $\mathrm{e}^{7,8}$. It is to be noted that the concentration of FDT used here is lower than previous report. ${ }^{8}$ Despite the PCE improvement of PSCs using such additives, their usage is incompatible with cost-effective processing (such as printing) and the possibility of reversible doping effect during prolonged operative conditions.

In this report, by substituting the problematic lithium with an asymmetric organic cations we exploited the usage of 1-butyl-3-methylpyridinium bis(trifluoromethylsufonyl)imide (BMPyTFSI) as a hydrophobic dopant for FDT as HTM in PSCs, systematically identify the mechanism of doping process and PV performances. 


\section{Results and discussion}

(a)
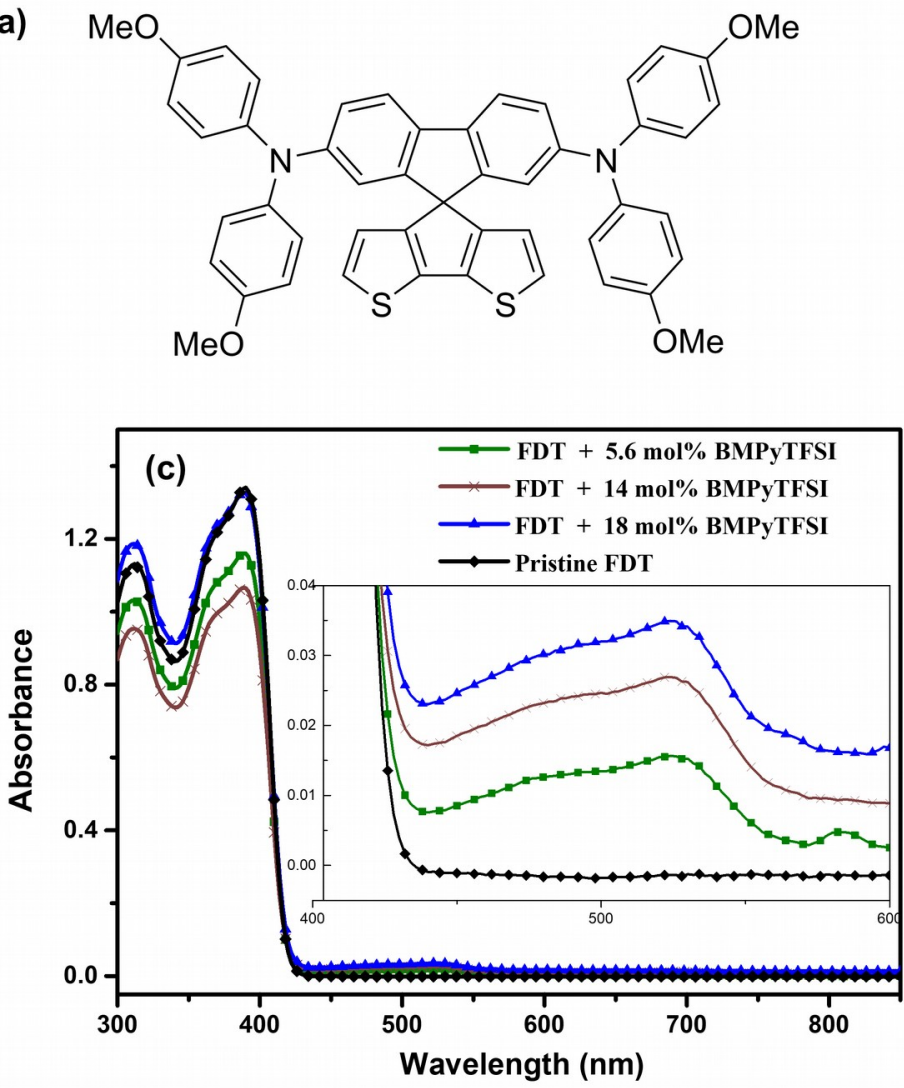

(b)<smiles>CCCC[n+]1cccc(C)c1</smiles>

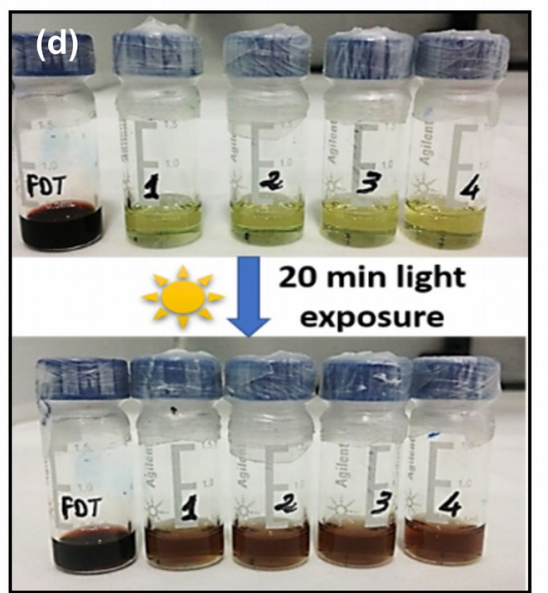

Figure 1. Chemical structure of a) FDT, b) 1-butyl-3-methylpyridinium bis(trifluoromethylsulfonyl)imide (BMPyTFSI), c) UV-Vis absorption spectra of FDT with different concentration of the hydrophobic dopant BMPyTFSI. The inset shows the enlarged spectra of the oxidized FDT peak at around $530 \mathrm{~nm}$ and d) FDT solution before and after 20min light exposure to 1 sun illumination.

The TFSI anion in BMPyTFSI has two strong electron withdrawing $\mathrm{CF}_{3} \mathrm{SO}_{2}-$ groups connected with $\mathrm{N}$, while the BMPy cation consists of one pyridinium group connected with butyl and methyl alkyl chains (Figure $1 b)^{19,20}$. The presence of $\mathrm{CF}_{3} \mathrm{SO}_{2}$ - groups in TFSI reduces the basic character of $-\mathrm{N}^{-}$, by extending the negative charge over multiple atoms, consequently promoting stability of the BMPy counter cation ${ }^{11,21-23}$. As depicted in Figure 1c, we investigated the absorption behavior of pristine FDT and doped FDT dissolved in chlorobenzene (CB) with different molar concentrations of BMPyTFSI. The BMPyTFSIdoped solutions, as well as the undoped FDT solutions, were illuminated under white light during 20 minutes. The inset in Figure1c shows the zoomed region around $530 \mathrm{~nm}$ peak 
showing partially oxidized FDT. Figure 1d shows a visual picture acquired for the FDT classically doped and with varying amount of BMPyTFSI (vial 1 - 4) before and after 20 min light exposure to 1 sun illumination showing the evident color change of the solution suggesting the completion of oxidation process.
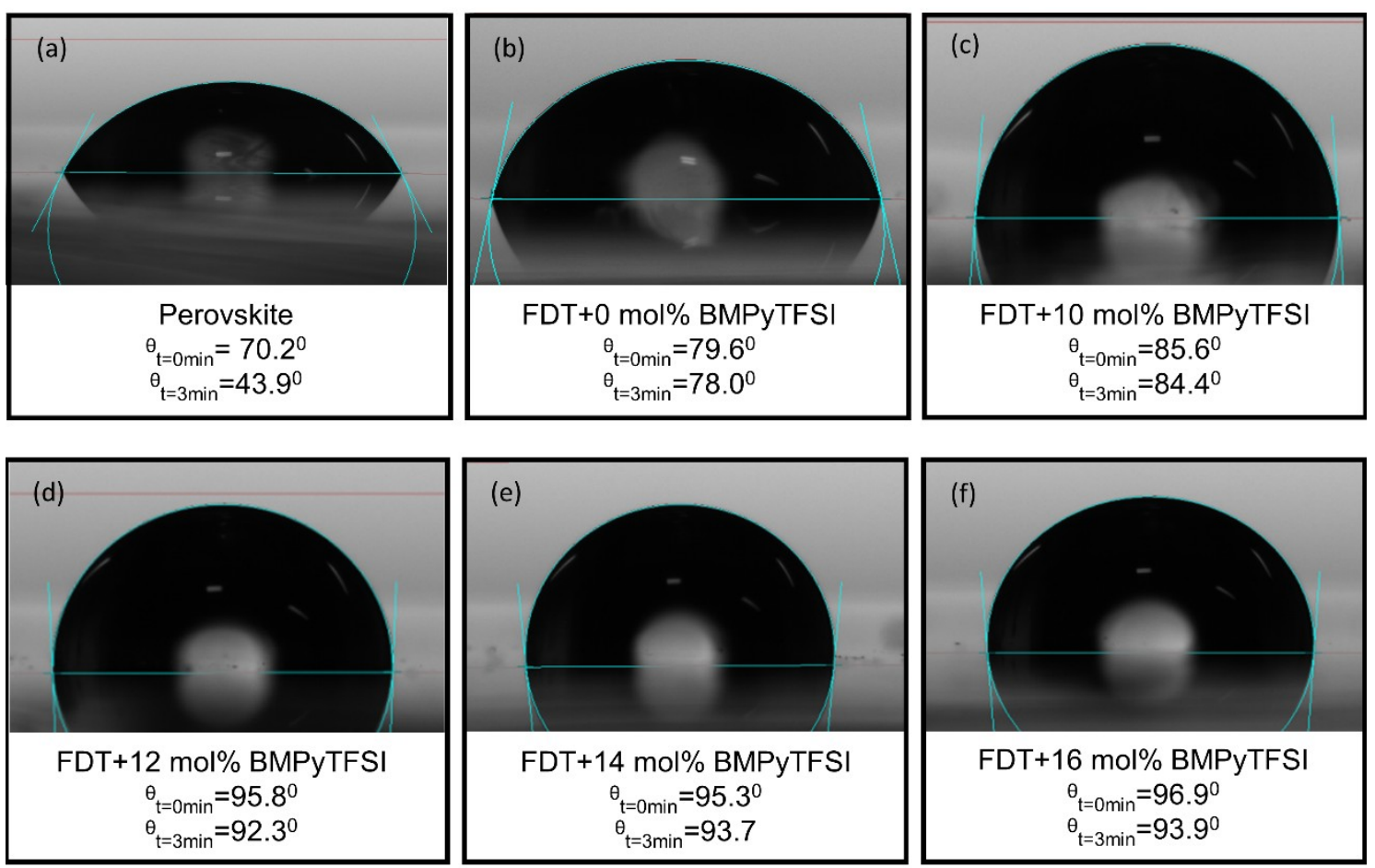

Figure 2. Contact angle $(\theta)$ measurements of a) perovskite b) and with FDT, with different BMPyTFSI dopant concentrations, c) $10 \mathrm{~mol} \%$, d) $12 \mathrm{~mol} \%$, e) $14 \mathrm{~mol} \%$ and f) $16 \mathrm{~mol} \%$.

To probe the surface hydrophobicity achieved by employing BMPyTFSI, contact angle $(\theta)$ measurements on FDT doped with varying concentrations of BMPyTFSI was undertaken. The resulted angle is the average of three different measurements, and each measurement was made at $\mathrm{t}=0 \mathrm{~min}$, while dripping the water droplet and after $3 \mathrm{~min}$ from the drop fall. The incident angle of the FDT-based HTM in its pristine form is $<80^{\circ}$, while this value increases linearly with the increase in concentration of BMPyTFSI, from $85.6^{\circ}$ for $10 \mathrm{~mol} \%$ of BMPyTFSI to $97^{\circ}$ for 16 mol\% of BMPyTFSI doping. We noted the contact angle value remains stable with time as of undoped HTM layers. The attended hydrophobicity conferred to the FDT-based hole transporting layers doped with BMPyTFSI will allow to achieve improved long-term stability of the underlying perovskite layer and protect it from moisture absorption. 
Table 1. Photovoltaics parameters extracted from $J-V$ curve for the champion cell under 100 $\mathrm{mW} / \mathrm{cm}^{2}$.

\begin{tabular}{lllll} 
HTM composition & $\boldsymbol{V}_{\mathbf{O C}}(\mathbf{m V})$ & $\boldsymbol{J}_{\text {SC }}\left(\mathbf{m A} \cdot \mathbf{c m}^{-2}\right)$ & $\mathbf{F F}(\mathbf{\%})$ & PCE (\%) \\
\hline FDT & 879 & 12.33 & 27.89 & 3.025 \\
\hline FDT + 10\% BMPyTFSI & 959 & 23.14 & 0.687 & 15.26 \\
\hline FDT + 14\% BMPyTFSI & 959 & 22.94 & 0.657 & 14.47 \\
\hline FDT + 18\% BMPyTFSI & 946 & 22.86 & 0.695 & 15.06 \\
\hline $\begin{array}{l}\text { FDT + 50mol\% LiTFSI + } \\
\text { 330mol\% t-BP + 10mol\% }\end{array}$ & 1074 & 22.95 & 0.74 & 18.24 \\
FK209 & & & & \\
\hline
\end{tabular}

The devices fabricated with pristine FDT showed PCE of 3\%, likely due to a high resistance registered for poor hole injecting properties of the undoped material that in turn promotes higher recombination. It can be deduced from the results shown in Table 1 that the PV parameters of the BMPyTFSI-doped devices are effectively enhanced with respect to pristine FDT, and that they are influenced by the amount of added dopant. Indeed, upon increasing the BMPyTFSI concentration over $10 \mathrm{~mol} \%$ resulted in a drop of performances, which can be due to the formation of inhomogeneous HTM layers. The conventional doped FDT-based devices, i.e. with LiTFSI, $t$-BP and FK209, yielded a PCE of 18.24\%. Thus, by simply utilizing the optimum molar percentage of BMPyTFSI, efficiency of about $\sim 85 \%$ of the conventional doped device can be obtained, without using hygroscopic materials that causes devices instability. The minute amount of BMPyTFSI dopant required as compared to high concentrations of dopants used in conventional devices is also remarkable. Fig 3a depicts the improved short circuit current value achieved in ionic liquid based devices, while voltage deficit was also noted. The voltage deficit was speculated to be due to band edge shifting, which can be minimized with further optimization. At 10 mol\% BMPyTFSI, we obtained a device with an efficiency of $15.26 \%$, which gave a $V_{\mathrm{OC}}$ of $959 \mathrm{mV}, J_{\mathrm{SC}}$ of $23.14 \mathrm{~mA} \mathrm{~cm}^{-2}$ and a fill factor (FF) of 68.7\% (Fig. 3a). Fig. 3b represents the external quantum efficiency (EQE) graphs, and expectedly the best performance was shown by conventional doped FDT, followed by ionic liquid based dopant with minor spectral losses. Fig.S1 represents the normalized efficiency at maximum power point tracking for a period of time, and BMPyTFSI based devices showed improved steady state performance as compared to conventional doped FDT. Fig.S2 represents the environmental stability of un-encapsulated devices in atmospheric conditions, where ionic liquid doped devices showed competitive performance. 

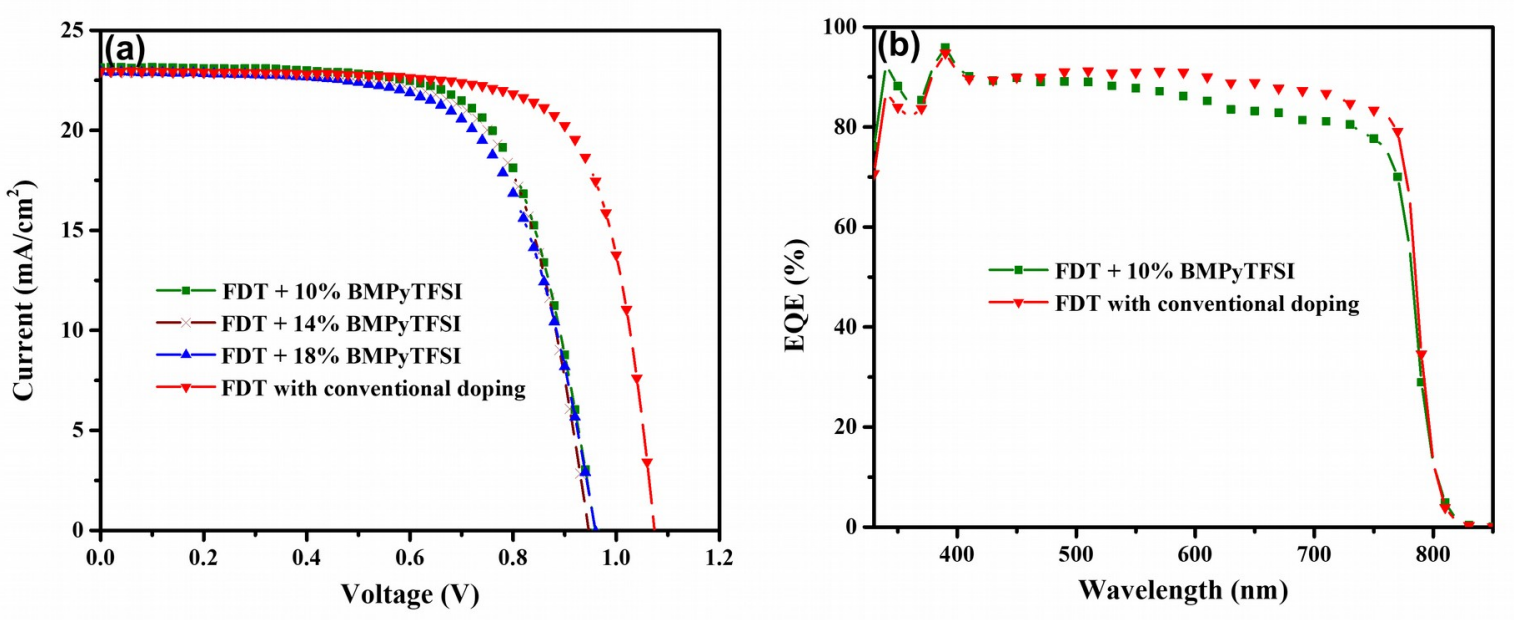

Figure 3. a) $J-V$ curves of the fabricated solar cells employing 10, 14, 18\% BMPyTFSI and conventional doped FDT (as reference) and b) IPCE under AM 1.5 illumination.

To derive the charge-transfer process in the fabricated PSCs, we performed impedance spectroscopy (IS). Figure 4a displays the recombination resistance vs voltage, while Figure 4b illustrates capacitance vs voltage extracted from Nyquist plots (Fig S3a), which depicts two irregular arcs, typical from complex triple-cation based PSCs. Therefore, an equivalent circuit model of one or two-RC elements were used in the analysis. In achieve better fitting, capacitor element was replaced by constant phase element (CPE), the CPE exponent was kept quite closely to the perfect capacitor value, $\mathrm{p} \sim 1$. From this graph, we noted the recombination resistance has similar slope, independently of the type of dopant used, indicating that the main contribution of recombination processes are in the bulk of the perovskite material. In a previous report ${ }^{24}$, a decrease in the carrier diffusion length (as for the case of FDT+BMPyTFSI) will intensify the effect of interfacial and bulk recombination. The capacitance behaviour of the devices was in accordance with the previous literature ${ }^{25}$ where the geometric capacitance value remains constant with voltage. Additionally, they follow the similar trend as of $J-V$ measurements, where 14 mol\% BMPyTFSI, shows a higher capacitance, indicating that higher charge concentration will take part in the interfacial recombination process. Correspondingly, a wide peak was obtained in the Bode plots (Fig. S3b), pointing several other processes are simultaneously taking place. As the low-frequency region is always associated with slow charge dynamic processes such as hysteresis, Figure $\mathrm{S} 4$, represents the capacitance versus frequency of the different samples at $V_{\mathrm{OC}}$ and suggest comparable device performance. 

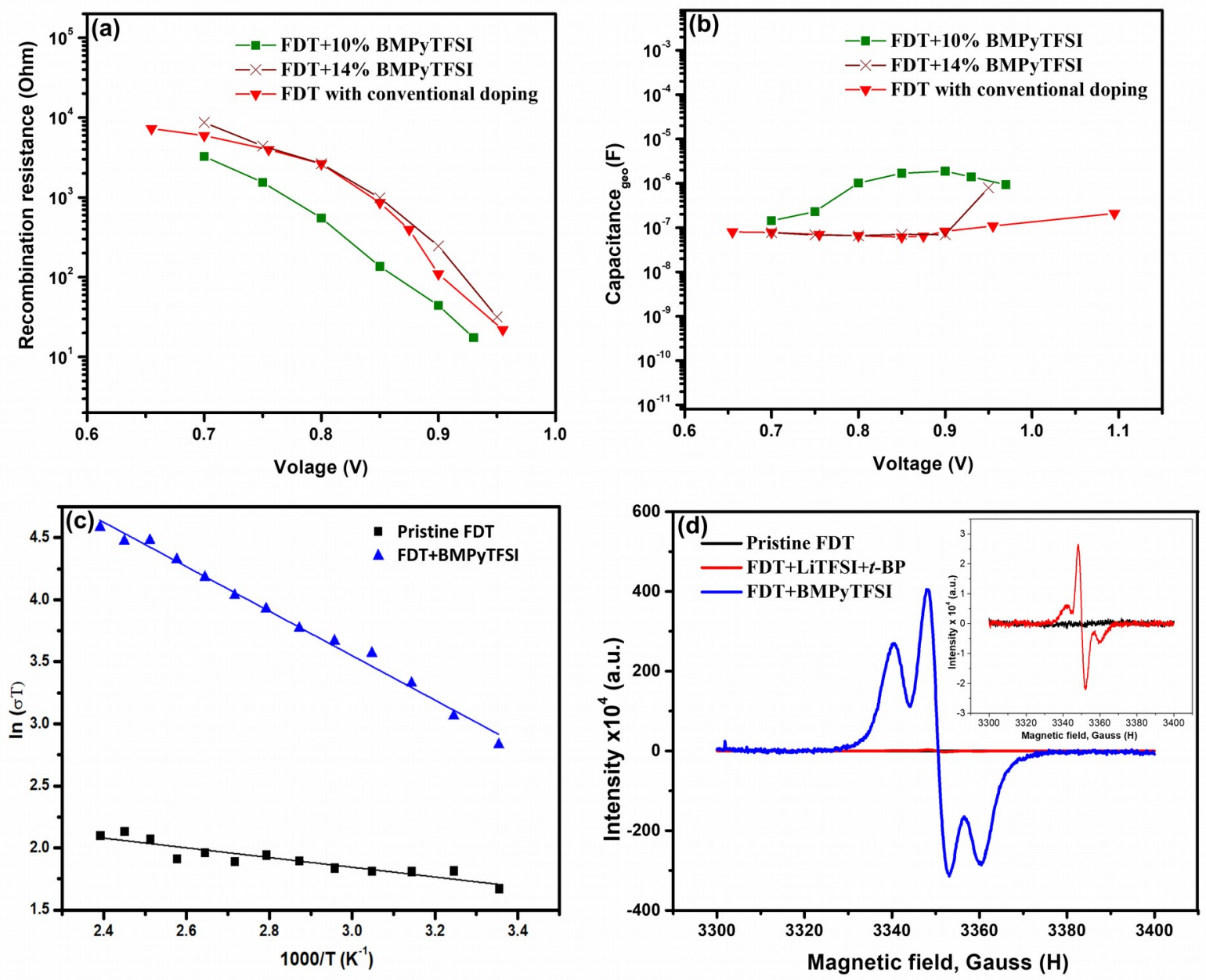

Figure 4. a) Recombination resistance and b) capacitance vs voltage extracted from impedance spectroscopy, c) temperature dependence conductivity and d) EPR spectra of FDT doped with different dopant.

Similar to any material which shows both extrinsic defects arising due to fabrication process errors and intrinsic defects due to intrinsic properties of materials. These intrinsic defects are sensitive to thermal conditions and effect electrical conductivity of materials. We evaluated the temperature-dependent electrical conductivity as a function of temperature and activation energy for ion migration according to the following Arrhenius relation given by ${ }^{26-28}$,

$$
\sigma(T)=\frac{\sigma_{0}}{T} \exp \left(\frac{-E_{a}}{K_{b} T}\right)
$$

where $\sigma(T)$ is the conductivity at given absolute temperature $\mathrm{T}, \quad \sigma \quad 0$ is the preexponential constant that depends on the vibrational frequency of the atoms and some structural parameters, $E_{\mathrm{a}}$ is the activation energy and $K_{\mathrm{b}}$ is the Boltzmann's constant. Figure 
4c represents the temperature-dependent conductivity for the pristine FDT and doped FDT. At elevated temperature $(>310 \mathrm{~K})$, the intrinsic defects start to get activated, resulting in the ionic conduction contribution commences to dominate in the total conductivity value ${ }^{26,28}$. The activation energy related to intrinsic defects is relatively higher and increases with temperature. The conductivity values increase with temperature in all the cases, however, FDT doped with BMPyTFSI shows significant conductivity improvement with temperature, suggesting the high thermal activation and contributed to higher $J_{\text {sc }}$ value.

To complement the electrical measurements and to study the doping effect, electron paramagnetic resonance (EPR) spectroscopy experiments were made on three different types of samples. Pristine FDT yielded a very weak signal, which was buried in the main spectra (inset Fig.4d), however BMPyTFSI doped FDT exhibit an intense signal (Figure 4d) that can be compared to classically doped FDT represented in inset. In both cases, the observed spectrum results from the superposition of two contributions, one of them with hyperfine structure originated by a ${ }^{14} \mathrm{~N}$-nucleus ( $\mathrm{I}=1$ ) and the other one a simple isotropic line. The spectra were evaluated with the Bruker-SpinFit program yielding g values, hyperfine couplings and line-widths (Fig. S5, Table 2). It is to note that due to the relative large adjustable parameters and significant correlation between line-widths and hyperfine couplings, the calculated values for these parameters can be not completely accurate. Moreover, the line-widths depend on concentration of paramagnetic species and the density of the medium. Taking this into account, it can be concluded that the radical species observed in both systems are the same.

Table 2. EPR spectral parameters for FDT+BMPyTFSI and FDT+LiTFSI+t-BP samples

\begin{tabular}{lll} 
Spin parameters & FDT+BMPyTFSI & FDT+LiTFSI+t-BP \\
\hline $\mathbf{g}_{1}$ & 2.0034 & 2.0033 \\
$\mathbf{\Delta B}_{\mathbf{1}}(\mathbf{G})$ & 5.6 & 6.6 \\
\hline $\mathbf{A}_{\mathbf{1}}(\mathbf{G})$ & 7.5 & 6.3 \\
$\mathbf{g}_{2}$ & 2.0031 & 2.0035 \\
\hline
\end{tabular}

$\begin{array}{lll}\Delta \mathbf{B}_{2}(\mathrm{G}) & 6.5 & 3.8\end{array}$


From the detection of a slight asymmetry on the bands and an irregular power saturation profile, authors ${ }^{11}$ suggested that two paramagnetic species, Spiro-OMeTAD ${ }^{+}$and SpiroOMeTAD-NH ${ }^{\circ}$, were presented in polymerizing ionic liquid doped Spiro-OMeTAD. According to this, in the present case the observation of a signal with ${ }^{14} \mathrm{~N}$ hyperfine splitting together with an exchange narrowed line confirms the simultaneous formation of $\mathrm{FAD}^{+}$and FAD- $\mathrm{NH}^{\mathrm{o}}$ radical cations. Arguably, it can be concluded that BMPyTFSI promotes the formation of oxidized species of FDT and will facilitate fast charge transfer. Furthermore, we have performed ${ }^{1} \mathrm{H}$-NMR spectroscopy measurements for the types of FDT in conventional and BMPyTFSI doped ones. FDT shows sharp and distinct peaks, recorded along with the characteristic FDT peak of 6.9-6.7 (fluorene) and 7.5 (Ph), however in the case of BMPyTFSI doped FDT broadening of such peaks as well as rise in peak intensity was noted. (Figure S6). The broadening of peaks may depicts environments exchange or H-bond formation, or due to the increase in concentration of $\mathrm{FAD}^{+}$and $\mathrm{FAD}-\mathrm{NH}^{\circ}$ species. We have also noted downward shifting for $\mathrm{BMPy}^{+}$with FDT, potentially due to opening of charge transfer process (oxidation) between $\mathrm{BMPy}^{+}$to ground state of FDT.

The characteristic feature of F1s peak in X-ray photoelectron spectroscopy (XPS) (Figure 5a) could be observed in conventional and BMPyTFSI systems at 689.2 and $689.1 \mathrm{eV}$ respectively due to presence of TFSI in each case $\mathrm{e}^{29,30}$. However, LiTFSI in combination with $t$-BP has the ability to improve the atomic percentage of $\mathrm{N}$ in the final FDT compared to the BMPyTFSI. Attributing to BMPyTFSI in FDT, a significantly high $\mathrm{F}$ and $\mathrm{O}$ atomic percentage were observed and the air exposure has a significant influence on the variation of oxygen atomic percentage in the sample ${ }^{30}$. In the high-resolution XPS analysis of C1s (Figure $5 b)$, the high oxidative-shifted carbon $\left(-\mathrm{C}^{-\mathrm{F}_{3}}\right)$ which is characteristic signature of TFSI ${ }^{29-31}$ was observed in both FDT+LiTFSI+t-BP and FDT+BMPyTFSI in which the peak intensity was significantly high for the later one.

The C-O- aromatic group associated with high binding energy and low binding energy C-O chemical state ${ }^{32}$ for FDT+BMPyTFSI show a narrow shift towards the low binding energy state compared to the other two systems (Figure 6a). Further, the organic C-O chemical state in $\mathrm{O} 1 \mathrm{~s}$ spectrum was observed at $531.3 \mathrm{eV}$ with $0.4 \mathrm{eV}$ chemical shift concerning the pristine FDT, probably due to the possibility of making $\mathrm{O}-\mathrm{F}_{\mathrm{x}}{ }^{30}$. Two un-ambiguous peaks were detected in the N1s spectrum for FDT+ BMPyTFSI (Figure S7), one at $399.7 \mathrm{eV}$ representing $\mathrm{C}-\mathrm{N}$ group in FDT and the other at $402.7 \mathrm{eV}$, which is assigned to the imide group of 
BMPyTFSI $^{32,33}$. Two additional peaks in S2p high resolution (Figure S8), XPS have emerged in doped FDT systems, one centred at 169.1 and the other at around $170.4 \mathrm{eV}$, which are assigned to $\mathrm{S} 2 \mathrm{p}_{3 / 2}$ and $\mathrm{S} 2 \mathrm{p}_{1 / 2}$ due to the presence of $-\mathrm{O}=\mathrm{S}=\mathrm{O}$ - group of the $\operatorname{LiTFSI}^{30,33}$.
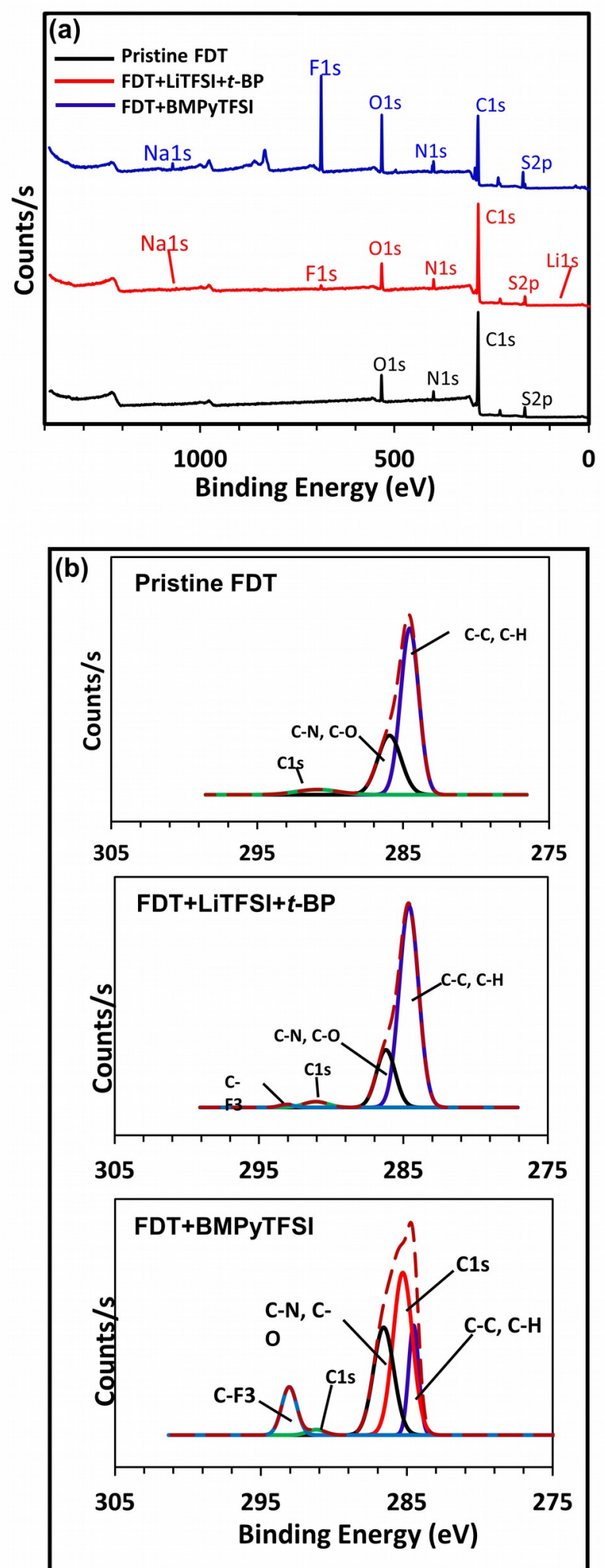
Figure 5. X-ray photoelectron spectroscopy (XPS), a) wide scan and b) high resolution C1s of FDT, FDT+LiTFSI+t-BP and FDT+BMPyTFSI.
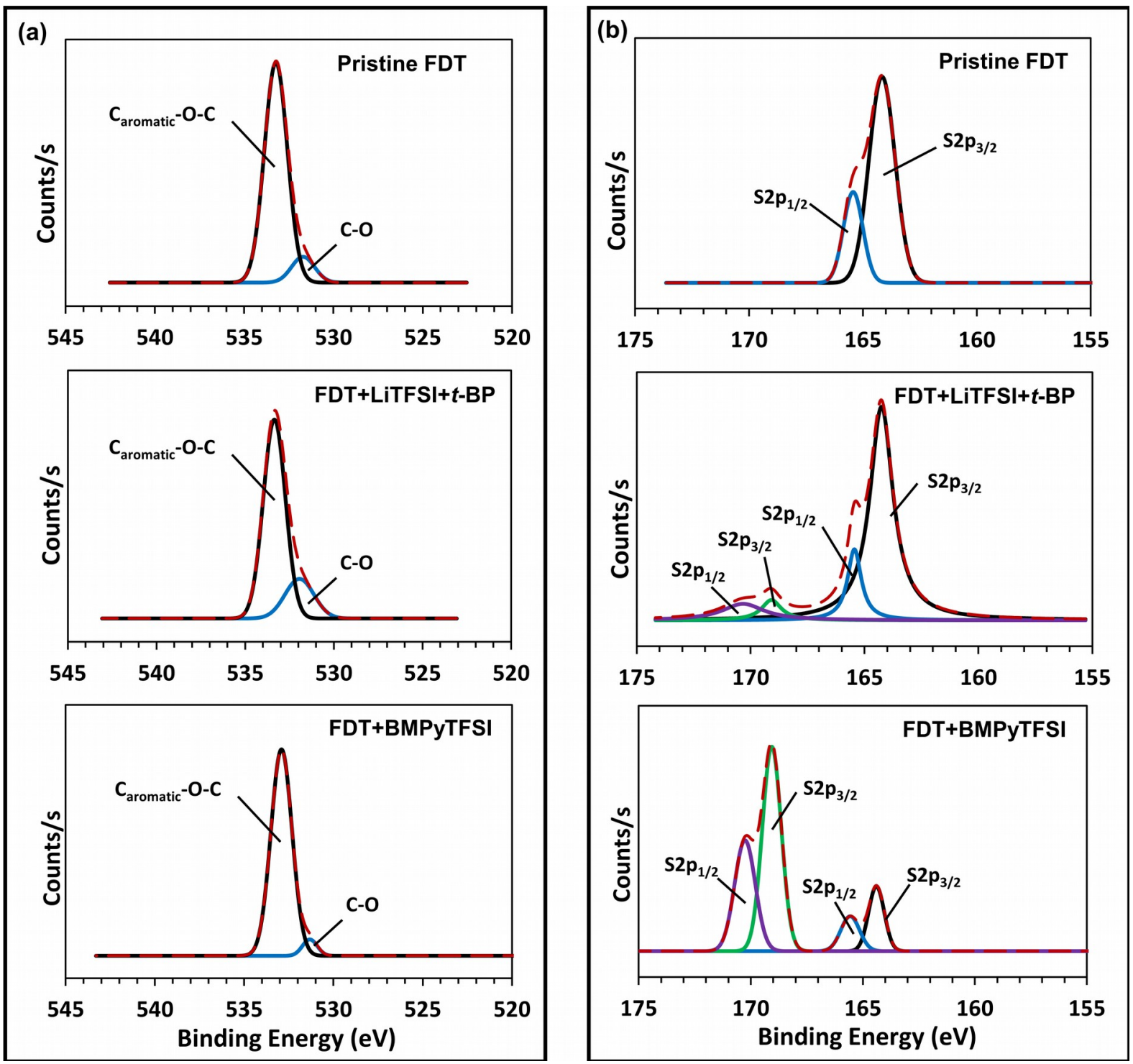

Figure 6. High resolution XPS of a) O1s and b) S2p of FDT, FDT+LiTFSI+t-BP and FDT+BMPyTFSI.

\section{Conclusions}

The usage of an ionic liquid based hydrophobic dopant, BMPyTFSI for FDT was demonstrated which function on par with a classical three components mixture of hygroscopic dopants. The charge transport and surface properties of FDT were studied and showed significant advantages by the use of ionic liquid dopant. The spectroscopic and electro-optical properties suggest the oxidation process occurred in FDT and the formation of 
the radical cation $\left(\mathrm{FAD}^{+}\right.$and $\left.\mathrm{FAD}-\mathrm{NH}^{\circ}\right)$ with increasing carrier concentration. The doping concentration was varied and its role on the photovoltaic parameters was evaluated. The XPS study reveals that identical characteristic elements belonging to TFSI, $t$-BP and BMPy in FDT, in which considerably high $\mathrm{F}$ and $\mathrm{O}$ atomic percentages were observed in BMPyTFSI doped system. Due to the possibility of making O-F in BMPyTFSI doped FDT, organic C-O chemical state in O1s spectrum showed $0.4 \mathrm{eV}$ chemical shift concerning to the pristine FDT. The BMPyTFSI doped devices will allow achieving improve stability and the usage of such a low doping concentration allowed to achieve similar performance in the fabricated devices.

\section{EXPERIMENTAL PROCEDURE}

Material and Methods

The chemicals were procured from Sigma Aldrich or Agros and were employed as such. FDT was synthesized according to previous reported method, ${ }^{8}$ while methylamine iodide, $\mathrm{CH}_{3} \mathrm{NH}_{3} \mathrm{I}$ (MAI) and formamidinium iodide (FAI), was purchased from Dyesol while $\mathrm{PbI}_{2}$, CsI was obtained from (TCI). 1-butyl-3-methylpyridinium bis(trifluoromethylsulfonyl)imide (BMPyTFSI) was procured from Ionic Liquids Technologies GmbH (IoLiTec).

Device fabrication:

Perovskite solar cells (PSCs) were fabricated on FTO-coated glass (TEC, Pilkington) patterned by laser etching. Prior to usage, the substrates were cleaned using Hellmanex solution and rinsed with deionized water and ethanol. Followed this they were ultrasonicated in acetone, washed using ethanol and 2-propanol and dried by using compressed air. $\mathrm{TiO}_{2}$ compact layer was then deposited using spray pyrolysis at $450{ }^{\circ} \mathrm{C}$ employing $1 \mathrm{~mL}$ of titanium diisopropoxide bis(acetyl acetonate) precursor solution (75 \% in 2-propanol) in $19 \mathrm{~mL}$ of pure ethanol using oxygen as carrier gas. Followed by these, the electrodes were kept for another 30 minutes at $450{ }^{\circ} \mathrm{C}$ to acquire anatase phase. Once the samples cooled down to room temperature, they $\mathrm{TiCl}_{4}$ treatment was made (by dipping in a $0.02 \mathrm{M} \mathrm{TiCl}_{4}$ solution in deionized water at $70^{\circ} \mathrm{C}$ for 30 minutes) to obtain a homogenous layer. Afterward, the samples were washed with deionized water, burned at $500^{\circ} \mathrm{C}$ for 10 minutes and left to cooled down to room temperature. $\mathrm{TiO}_{2}$ mesoporous layer (Dyesol, 30NRD) was then spin coated (4000rpm at $2000 \mathrm{rpm} /$ seconds as acceleration for 30s) and the samples were annealed by heating them up progressively to $450{ }^{\circ} \mathrm{C}$ for 2 hours.

Perovskite thin films with an composition of $\mathrm{Cs}_{0.1}\left(\mathrm{FAPbI}_{3}\right)_{0.81}\left(\mathrm{MAPbBr}_{3}\right)_{0.09}$ were prepared from a perovskite precursor solution containing CsI (0.13 M), FAI (1.05 M), $\mathrm{PbI}_{2}(1.24 \mathrm{M})$, MABr $(0.12 \mathrm{M})$ and $\mathrm{PbBr}_{2}(0.12 \mathrm{M})$ in a anhydrous solvent mixture of $N, N$ - 
dimethylformamide (DMF) and dimethylsulfoxide $(\mathrm{DMSO})=4: 1$ (volume ratio). The triple cation perovskite solution was spun on the electrode in a two-step spin-coating program set as 2000 and $5000 \mathrm{rpm}$ for 10 and $30 \mathrm{~s}$, respectively. During the spin process, $100 \mu \mathrm{L}$ of chlorobenzene was dripped on the substrate in the last $10 \mathrm{~s}$ of termination time of spinner. The electrodes were left to annealed at $100{ }^{\circ} \mathrm{C}$ for $1 \mathrm{~h}$ for perovskite crystallization.

HTM preparation

FDT solutions were prepared by dissolving $50 \mathrm{mM}$ in chlorobenzene. 1-butyl-3methylpyridinium bis(trifluoromethylsulfonyl)imide (BMPyTFSI) was used as a dopant as well as additives for FDT. The stock solution of BMPyTFSI was prepared by adding the mole $\%$ in acetonitrile. For comparison purpose, the doped FDT solution was also prepared by dissolving $50 \mathrm{mM}$ in chlorobenzene, plus regular additives i.e. $17.5 \mu \mathrm{L}$ of a lithium bis (trifluoromethylsulphonyl)imide (LiTFSI) stock solution (520 mg of LiTFSI in $1 \mathrm{~mL}$ of acetonitrile), $21.9 \mu \mathrm{L}$ of a FK209 (Tris(2-(1H -pyrazol-1-yl)-4-tert-butylpyridine)cobalt(III)Tris(bis(trifluoro-methylsulfonyl)imide))) stock solution (400 mg in $1 \mathrm{~mL}$ of acetonitrile) and $28.8 \mu \mathrm{L}$ of 4 -tert-butylpyridine $(t-B P)$. Then $35 \mu \mathrm{L}$ of HTM solution were dropped on the perovskite substrates and were spin coated at $4000 \mathrm{rpm}$ for 30 seconds in both the cases. Following this, $80 \mathrm{~nm}$ of gold was used as cathode which was thermally evaporated atop of FDT under a vacuum level between $1 \times 10^{-6}$ and $1 \times 10^{-5}$ torr.

\section{Characterization}

To record absorption spectra FDT was dissolved in chlorobenzene and were acquired using 1 cm cuvette with various concentration of BMPyTFSI. The samples for conductivity measurements was made on interdigitated electrodes from room temperature to $150{ }^{\circ} \mathrm{C}$ using Linkam sample holder (parallel method) connected to a source meter and LabVIEW was used for data acquisition. Electron Paramagnetic Resonance (EPR) spectra were recorded on a Bruker ELEXSYS 500 spectrometer (X band) at room temperature. The spectrometer was equipped with a superhigh-Q resonator ER-4123-SHQ and solution samples were placed in quartz tubes. The magnetic field was calibrated by a NMR probe and the frequency inside the cavity ( 9.395 GHz) was determined with an integrated MW-frequency counter. X-ray photoelectron spectroscopy (XPS) experiments were carried out on a SPECS system (Berlin, Germany) equipped with Phoibos 150 1D-DLD analyzer with monochromated $\mathrm{Al} \mathrm{K}_{\alpha}$ radiation $(1486.7 \mathrm{eV})$. The wide scan was performed with the step energy of $1 \mathrm{eV}$ (dwell time: $0.1 \mathrm{~s}$, pass energy: $80 \mathrm{eV}$ ), and detailed analysis of the elements was performed using $0.08 \mathrm{eV}$ step energy (dwell time: $0.1 \mathrm{~s}$, pass energy: $30 \mathrm{eV}$ ) with an electron exit angle of $90^{\circ}$. 
The spectra was adjusted using CasaXPS 2.3.16 software, which models Gauss-Lorentzian contributions.

The NMR experiments were carried out on a Bruker AV500 spectrometer, equipped with a 5 $\mathrm{mm} \mathrm{BBI}$ probe and gradients on the $\mathrm{Z}$ axis, operating at a frequency of $500 \mathrm{MHz}$ for proton using DMSO- $\mathrm{d}_{6}$ as solvent. Data acquisition and data processing was carried out with the TOPSPIN 2.1 software (Bruker). The pulse sequences used were the Bruker standard. The chemical shifts have been referenced with respect to the residual solvent signal.

Current density-voltage $(J-V)$ curves were recorded by applying an external potential bias to the devices and the generated photocurrent was recorded at scan rate of $10 \mathrm{mV} / \mathrm{s}$ with the help of Keithley 2400 source meter. The light source was a $450 \mathrm{~W}$ xenon lamp (Oriel) equipped with a SchottK113 Tempax sunlight filter (Praezisions Glas \& Optik GmbH) to match the emission spectrum of the lamp to the AM1.5G standard. The light intensity was determined using a calibrated Si reference diode equipped with an infrared cutoff filter (KG3, Schott). IPCE spectra were recorded as a function of wavelength under a constant white light bias of $\approx 10 \mathrm{~mW} \mathrm{~cm}^{-2}$ radiated by an array of white light emitting diodes. The excitation beam from a $300 \mathrm{~W}$ xenon lamp (ILC Technology) was focused through a Gemini-180 double monochromator (Jobin Yvon Ltd) and chopped at $\approx 2 \mathrm{~Hz}$. The signal was recorded using a Model SR830 DSP Lock-In Amplifier (Stanford Research Systems). The measurements were conducted using a black mask with an aperture area $0.16 \mathrm{~cm}^{2}$ to define the active area of the device. Electrochemical Impedance spectroscopy (EIS) studies were made with the help of Bio-logic (SP-300), under illumination of a warm white LED over a range of DC light intensities. Frequency response of the solar cells at different positions of the Fermi level, was fixed by the DC illumination intensity (open circuit voltage) to avoid voltage drop. A $20 \mathrm{mV}$ perturbation step in the range $2 \mathrm{MHz}-1 \mathrm{mHz}$ was used to obtain the spectra. Following the measurements, the data were fitted with the help of Z-view software to extract the characteristic parameters of the devices.

\section{Supplementary Information}

Supplemental information can be found with this article online at

\section{Acknowledgements}

This work has received funding from the European Union H2020 Programme under European Research council Consolidator grant [MOLEMAT, 726360], we gratefully acknowledge Advanced Research Facilities (SGIker) of the University of Basque Country / Euskal Herriko Unibertsitatea for 
NMR measurements and spectroscopy measurements. MSM thanks the National Research grant "Juan de la Cierva” [FJCI-2017-31761]. We thank Meenakshi Pegu for NMR experiments and Peng Huang for experimental help.

\section{Author Contributions}

NH collected the data and analysed XPS, SK performed electrical and spectroscopic measurements, LC contributed with absorbance spectroscopy and initial device fabrication, SP fabricated triple cation devices, MSM performed impedance spectroscopy, GP conceived and synthesized FDT molecule, LL performed EPR measurements and analysis, MKN supervised devices fabrication of SP. SA supervised and directed the research. All authors contributed to the draft and commented to the completion of the final document.

\section{Conflict of Interests}

The authors declare no competing interests.

\section{References:}

(1) Kim, H.-S.; Lee, C.-R.; Im, J.-H.; Lee, K.-B.; Moehl, T.; Marchioro, A.; Moon, S.-J.; Baker, R.H.; Yum, J.-H.; Moser, J.E.; et al. Lead Iodide Perovskite Sensitized All-Solid-State Submicron Thin Film Mesoscopic Solar Cell with Efficiency Exceeding 9\%. Sci. Rep. 2012, 2, 591 .

(2) Lee, J.-W.; Dai, Z.; Han, T.-H; Choi, C.; Chang, S.-Y.; Lee, S.-J.; Marco, N.D.; Zhao, H.; Sun, P.; Huang, Y.; et al. 2D Perovskite Stabilized Phase-Pure Formamidinium Perovskite Solar Cells. Nat. Commun. 2018, 9, 3021.

(3) Im, J.-H.; Lee, C.-R.; Lee, J.-W.; park, S.-W.; Park, N.-G. 6.5\% Efficient Perovskite Quantum-Dot-Sensitized Solar Cell. Nanoscale 2011, 3, 4088-4093.

(4) Yang, W.S.; Park, B.-W.; Jung, E.H.; Jeon, N.J.; Kim, Y.C.; Lee, D.U.; Shin, S.S.; Seo, J.; Kim, E.K.; Noh, J.H.; et al. Iodide Management in Formamidinium-Lead-Halide Based Perovskite Layers for Efficient Solar Cells. Science 2017, 356 (6345), 1376-1379. 
(5) Wang, D.; Wright, M.; Elumalai, N.K.; Uddin, A. Stability of Perovskite Solar Cells. Sol. Energy Mater. Sol. Cells 2016, 147, 255-275.

(6) Berhe, T. A.; Su, W.-N.; Chen, C.-H.; Pan, C.-J.; Cheng, J.-H.; Chen, H.-M.; Tsai, M.-C.; Chen, L.-Y.; Dubale, A.A.; Hwang, B.-J. Organometal Halide Perovskite Solar Cells: Degradation and Stability. Energy Environ. Sci. 2016, 9, 323-356.

(7) Calio, L.; Salado, M.; Kazim, S.; Ahmad, S. A Generic Route of Hydrophobic Doping in Hole Transporting Material to Increase Longevity of Perovskite Solar Cells. Joule 2018, 2(9), 1800-1815.

(8) Saliba, M.; Orlandi, S.; Matsui, T.; Aghazada, S.; Cavazzini, M.; Baena, J.-P.C.; Gao, P.; Scopelliti, R.; Mosconi, E.; Dahmen, K.-H.; et al. A Molecularly Engineered HoleTransporting Material for Efficient Perovskite Solar Cells. Nat Energy 2016, 1, 15017.

(9) Liu, T.; Sun, K.; He, R.; Zhang, Z.; Shen, W.; Li, M. How the Connectivity of Methoxy Substituents Influences the Photovoltaic Properties of Dissymmetric Core Materials: A Theoretical Study on FDT. J. Phys. Chem. C 2018, 122, 8804-8813.

(10) Zhang, H.; Shi, Y.; Yan, F.; Wang, L.; Wang, K.; Xing, Y.; Dong, Q.; Ma, T. A Dual Functional Additive for the HTM Layer in Perovskite Solar Cells. Chem. Commun. 2014, 50, 5020-5022.

(11) Abate, A.; Hollman, D.J.; Teuscher, J.; Pathak, S.; Avolio, R.; D’Errico, G.; Vitiello, G.; Fantacci, S.; Snaith, H.J. Protic Ionic Liquids as p-Dopant for Organic Hole Transporting Materials and Their Application in High Efficiency Hybrid Solar Cells. J. Am. Chem. Soc. 2013, 135 (36), 13538-13548.

(12) Arora, N.; Orlandi, S.; Dar, M.I.; Aghazada, S.; Jacopin, G.; Cavazzini, M.; Mosconi, E.; Gratia, P.; Angelis, F.D.; Pozzi, G. High Open-Circuit Voltage: Fabrication of Formamidinium Lead Bromide Perovskite Solar Cells Using Fluorene-Dithiophene Derivatives as Hole-Transporting Materials. ACS Energy Lett. 2016, 1, 1, 107-112.

(13) Torres, A.; Rego, L.G.C. Surface Effects and Adsorption of Methoxy Anchors on Hybrid Lead Iodide Perovskites: Insights for Spiro-MeOTAD Attachment. J. Phys. Chem. C 2014, $118,46,26947-26954$. 
(14) Yin, J.; Cortecchia, D.; Krishna, A.; Chen, S.; Mathews, N.; Grimsdale, A.C.; Soci, C. Interfacial Charge Transfer Anisotropy in Polycrystalline Lead Iodide Perovskite Films. J. Phys. Chem. Lett. 2015, 6, 8, 1396-1402.

(15) Pastore, M.; Mosconi, E.; Angelis, F.D. Computational Investigation of Dye-Iodine Interactions in Organic Dye-Sensitized Solar Cells. J. Phys. Chem. C 2012, 116, 9, 59655973.

(16) Cossi, M.; Rega, N.; Scalmani, G.; Barone, V. Energies, Structures, and Electronic Properties of Molecules in Solution with the C-Pcm Solvation Model. J. Comput. Chem. 2003, 24, 6, 669-681.

(17) Snaith, H.J.; Gratzel, M. Enhanced Charge Mobility in a Molecular Hole Transporter via Addition of Redox Inactive Ionic Dopant: Implication to Dye-Sensitized Solar Cells. Appl.Phys. Lett. 2006, 89, 262114.

(18) Abate, A.; Leijtens, T.; Pathak, S.; Teuscher, J.; Avolio, R.; Errico, M.E.; Kirkpatrik, J.; Ball, J.M; Docampo, P.; McPherson, I. Lithium Salts as “Redox Active” p-Type Dopants for Organic Semiconductors and Their Impact in Solid-State Dye-Sensitized Solar Cells. Phys. Chem. Chem. Phys. 2013, 15, 2572-2579.

(19) Ghosh, S.; Singh, T. Role of Ionic Liquids in Organic-Inorganic Metal Halide Perovskite Solar Cells Efficiency and Stability. Nano Energy 2019, 63, 103828.

(20) Schloemer, T.H.; Christians, J.A.; Luther, J.M.; Sellinger, A. Doping Strategies for Small Molecule Organic Hole-Transport Materials: Impacts on Perovskite Solar Cell Performance and Stability. Chem. Sci. 2019, 10, 1904-1935.

(21) Greaves, T.L.; Drummond, C.J. Protic Ionic Liquids: Properties and Applications. Chem. Rev. 2008, 108, 1, 206-237.

(22) Shi, J.; Chai, Z.; Tang, R.; Li, H.; Han, H.; Peng, T.; Li, Q.; Li, Z. Effect of ElectronWithdrawing Groups in Conjugated Bridges: Molecular Engineering of Organic Sensitizers for Dye-Sensitized Solar Cells. Front. Optoelectron 2016, 9, 1, 60-70. 
(23) Armand, M.; Endres, F.; MacFarlance, D.R, Ohno, H.; Scrosati, B. Ionic-Liquid Materials for the Electrochemical Challenges of the Future. Nat. Mater. 2009, 8, 621-629.

(24) Xiang, J.; Li, Y.; Huang, F.; Zhong, D. Effect of Interfacial Recombination, Bulk Recombination and Carrier Mobility on the J-V Hysteresis Behaviors of Perovskite Solar Cells: a Drift-Diffusion Simulation Study. Phys. Chem. Chem. Phys., 2019, 21, 17836-17845.

(25) Ebadi, F.; Taghavinia, N.; Mohammadpour, R.; Hagfeldt, A.; Tress, W. Origin of Apparent Light-Enhanced and Negative Capacitance in Perovskite Solar Cells. Nat Commun. 2019, 10, 1574.

(26) Lee, J.-W.; Kim, S.-G.; Yang, J.-M.; Yang, Y.; Park, N.-G. Verification and Mitigation of Ion Migration in Perovskite Solar Cells. APL Mater. 2019, 7, 041111.

(27) Xing, J.; Wang, Q.; Dong, Q.; Yuan, Y.; Fang, Y.; Huang, J. Ultrafast Ion Migration in Hybrid Perovskite Polycrystalline Thin Films Under Light and Suppression in Single Crystals. Phys. Chem. Chem. Phys. 2016, 18, 30484-30490.

(28) Li, H.; Wang, X.; Zhang, T.; Gong, X.; Sun, Q.; Pan, H.; Shen, Y.; Ahmad, S.; Wang, M. Layered Ruddlesden-Popper Efficient Perovskite Solar Cells with Controlled Quantum and Dielectric Confinement Introduced via Doping. Adv. Funct. Mater. 2019, 29 (30), 1903293.

(29) Dedryvere, R.; Leroy, S.; Martinez, H.; Blanchard, F.; Lemordant, D.; Gonbeau, D. XPS Valence Characterization of Lithium Salts as a Tool to Study Electrode/Electrolyte Interfaces of Li-Ion Batteries. J. Phys. Chem. B. 2006, 110 (26), 12986-12992.

(30) Hawash, Z.; Ono, L.K.; Raga, S.R.; Lee, M.V.; Qi, Y. Air-Exposure Induced Dopant Redistribution and Energy Level Shifts in Spin-Coated Spiro-OMeTAD Films. Chem. Mater. 2015, 27 (2), 562-569.

(31) Gilman, J. B.; Eliason, T.L.; Fast, A.; Vaida, V. Selectivity and Stability of Organic Films at the Air-Aqueous Interface. J. Colloid Interface Sci. 2004, 280 (1), 234-243. 
(32) Ono, L. K.; hawash, Z.; Perez, E.J.J.; Qiu, L.; Jiang, Y.; Qi, Y. The Influence of Secondary Solvents on the Morphology of a Spiro-MeOTAD Hole Transport Layer for Lead Halide Perovskite Solar Cells J. Phys. Chem. lett. 2018, 51 (29), 294001.

(33) Ensling, D.; Stjerndahl, M.; Nyten, A.; Glstafsson, T.; Thomas, J.O. A Comparative XPS Surface Study of $\mathrm{Li}_{2} \mathrm{FeSiO}_{4} / \mathrm{C}$ Cycled with LiTFSI- and $\mathrm{LiPF}_{6}$-Based Electrolytes J. Mater. Chem. 2009, 19 (1), 82-88. 


\section{TOC Graphic}

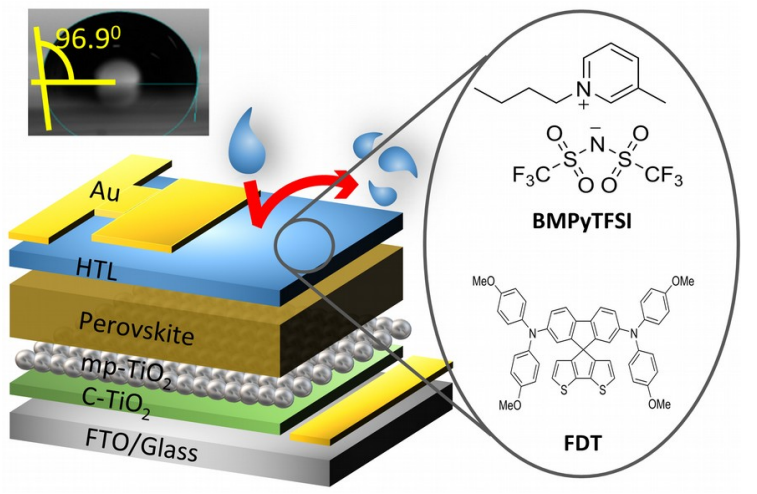


